\title{
The Ecosystem in Practice: Interest and Problems of an Old Definition for Constructing Ecological Models
}

\author{
Jacques Gignoux, ${ }^{1 *}$ Ian D. Davies, ${ }^{2}$ Shayne R. Flint, ${ }^{3}$ \\ and Jean-Daniel Zucker ${ }^{4,5}$
}

\begin{abstract}
${ }^{1}$ Biogéochimie et Écologie des Milieux Continentaux, CNRS UMR 7618, Ecole Normale Supérieure, 46 rue d'Ulm, 75230 Paris cedex 05, France; ${ }^{2}$ Fenner School of Environment and Society, ANU College of Medicine, Biology and Environment, The Australian National University, Canberra, ACT 0200, Australia; ${ }^{3}$ School of Computer Science, College of Engineering and Computer Science, The Australian National University, Canberra, ACT 0200, Australia; ${ }^{4}$ Unité de Modélisation Mathématique et Informatique des Systèmes Complexes, UMI 209, IRD France Nord, 32 av. Henri Varagnat, 93143 Bondy cedex, France; ${ }^{5}$ Cordeliers Research Center, UMR-S 872, Pierre and Marie Curie-Paris 6 University, 15 rue de l'Ecole de Médecine, 75006 Paris, France
\end{abstract}

\begin{abstract}
Since its inception, the ecosystem concept has been widely used in ecology and is increasingly finding application within other disciplines. In more recent times within ecology, however, it has been suggested the term is now obsolete. We argue that three problems lie at the heart of these criticisms, namely the physics-biology duality problem, the boundary problem and the abstraction problem. The physics-biology duality problem (how to grapple with systems that follow the laws of both physics and biology) is addressed by modern computer science techniques originating from simulation and software engineering. The boundary problem (how to find the limits of an ecosystem in the real world) is solved by a powerful assumption of Tansley, that the ecosystem is an ad hoc construct on the part of an observer for a particular purpose. The abstraction problem (can models of an ecosystem at different levels of detail produce the same
\end{abstract}

outcomes) has no general solution, but can be improved upon by using scaling techniques and standards to facilitate model comparisons. We demonstrate that Tansley's (Ecology 16:284-307, 1935) definition is still relevant to modern ecology almost as is. Tansley's ecosystem is a multi-disciplinary, recursive, scale-independent and observerdependent object. These properties closely match those of complex systems as defined in mathematics and computer sciences. From Tansley's definition, we propose a formal description of the concepts and relations linked to the ecosystem definition, as an ontology that can serve as a basis for future discussion, modelling and conceptual work.

Key words: ecosystem definition; ontology; complex system; hierarchy; landscape; abstraction.
Received 23 December 2010; accepted 5 July 2011; published online 7 September 2011

Electronic supplementary material: The online version of this article (doi:10.1007/s10021-011-9466-2) contains supplementary material, which is available to authorized users.

Author Contributions: All authors have had a significant input into the conceptual thinking involved in this paper, have contributed to the writing of the manuscript and design of the ecosystem ontology.

*Corresponding author; e-mail: gignoux@biologie.ens.fr

\section{INTRODUCTION}

Ecology is a synthetic science (Odum 1977) and draws from a wide range of disciplines. An important outcome arising from this synthesis has been the concept of the ecosystem which has in turn fertilized many other fields (for example, see Pickett and Cadenasso 2002). The term first appears in 
Tansley (1935) and despite its age is still often cited. According to the ISI web of knowledge ${ }^{\mathrm{SM}}$ database, half the citations have occurred over the past decade. The concept itself has become increasingly important. A keyword search reveals the term 'ecosystem' appears in $80 \%$ of those papers containing 'ecology' from 2005 onwards (Figure 1).

Pioneering works such as Lindeman (1942) recognized the importance of the concept and contributed to its success. In subsequent years, many attempts have been made at clarification (for example, Odum and Odum (1953); Allen and Hoekstra (1992); Pickett and Cadenasso (2002)). Some authors argue that the concept now poses problems that hamper progress of the science (O'Neill 2001). A thorough terminological analysis, however, reveals that this might well be due to the later additions to the definition (Jax 2007), mixing definition terms with descriptions frequently correlated to the concept. Additional confusion arises depending on the purpose of the study: modellers and field scientists view ecosystems differently (Allen and Hoekstra 1992), which perhaps has led to the uneasiness pointed to by $\mathrm{O}^{\prime} \mathrm{Neill}$ (2001). Jax (2007) even states that given the history of the

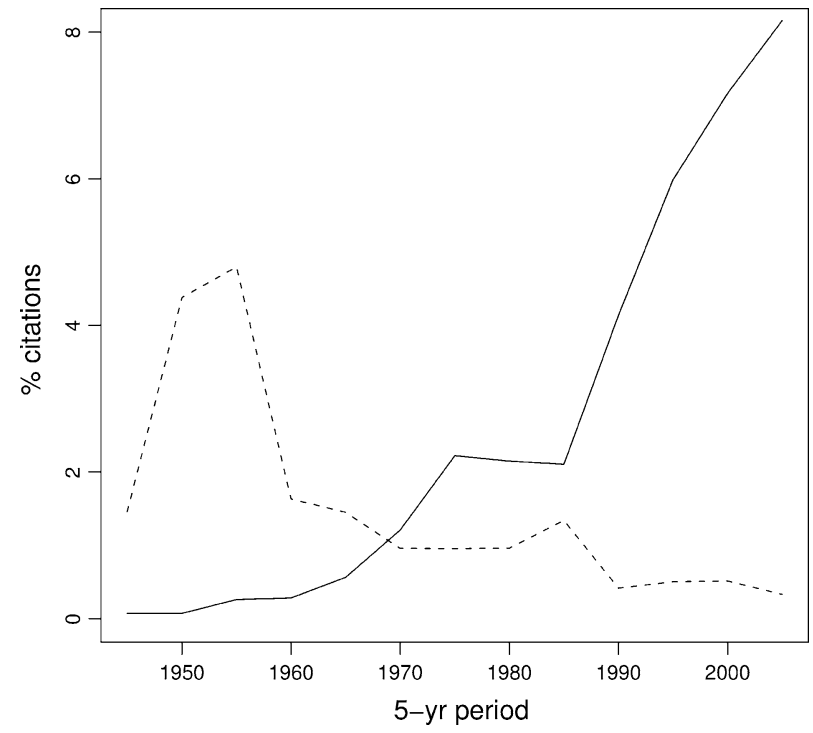

Figure 1. Citations of the original article defining the ecosystem (Tansley 1935) from the information of the ISI web of knowledge ${ }^{\mathrm{SM}}$ database. Solid lines ratio of the number of articles with the keyword "ecosystem" over the number of articles with the keyword "ecology" (/10, that is, in $200580 \%$ of ecology papers included the keyword "ecosystem"); dashed line \% of articles with the keyword "ecology" citing Tansley (1935). Citations by books not included, and probably many early citations missing. The number of publications including the "ecology" keyword increased exponentially over the period, with a 100-fold increase in 60 years. concept "ecosystem" [...] and the epistemological status of ecological units, there is not a single "right" definition for the term "ecosystem". There can be different useful definitions for different purposes'.

It is important that terms adapt to new knowledge. However, we face a problem in building a predictive science if terms remain ill-defined. Past debates can partly be attributed to different interpretations of the ecosystem concept (see the 'cybernetic ecosystem debate' in Jax (2007), problems defining stability in Pimm (1984); not to mention the remarkable fog surrounding the notion of 'ecosystem functioning' in the recent biodiversity debate (Jax 2005)). Attempting to reinvigorate the ecosystem concept is a challenging task given its long history (Jax 2007), and the high complexity of the objects and diversity of methods used in ecology. In a comprehensive analysis of the problem, Jax (2006) has proposed the construction of clusters of 'operational definitions' associated with their traditional 'generic' meanings necessary for heuristic discussion.

Our focus is modelling. Concepts are involved in all aspects of theoretical and applied modelling: Modellers use existing concepts in development, analyze concepts in synthetic modelling and model coupling, produce new concepts in theoretical modelling, and confront them with data when testing. It has been advocated that ecological modellers use standard methods to describe models in publications, to facilitate understanding and comparison: for example, Grimm and others (2006) defined the ODD protocol to describe individualand agent-based models, and later proposed (Grimm and others 2010) to extend its use to any type of large complex model. In thermodynamics, a unique definition of the thermodynamic system (Carnot 1824) is used to construct all kinds of models and experiments. This is made possible through common agreement on the system concept and the rigour defining the system under study. If the ecosystem is to play an analogous role, the multiple versions of the ecosystem definition resulting from its history are problematic. Ecological modelling not only requires a standard way of describing models, but also an accurate, commonly accepted, shared conceptual base upon which to build.

Building consistent sets of axiomatic definitions for any particular domain is now a scientific field per se. Such constructions or ontologies (Morin 1986; Gruber 1993; Guarino 1995) are an explicit specification of a conceptualization. Conceptualization means here an abstraction of the world that 
we wish to represent for some purpose, for example, to draw inferences and perform automatic classification. Ontologies explicitly specify concepts and their relations, and software exists to produce automatic analyses of the consistency of such constructs (Gómez-Pérez and others 2004; De Nicola and others 2009). Appendix S1 in Supplementary material lists a number of fields where ontologies have been developed.

Our goal in this article is to construct an ontology of the ecosystem and related concepts, beginning with the definition by Tansley which, we will argue, is already sufficiently rich. We will (1) analyze the main foundations of the concept, (2) examine problems linked to the definition, and (3) propose a modern interpretation, as an ontology, of the concepts involved in the ecosystem definition. We view this work as a counterpart to Grimm's ODD language of model description (Grimm and others 2006; Grimm and others 2010). We hope our initiative will foster the development of simulation or modelling platforms, within which model comparison would be greatly facilitated by the common conceptual base and potential that ontologies provide for the development of formal automated tools.

\section{The Richness of a 75-year-old Definition}

In the original definition of the ecosystem by Tansley (1935), the term does not appear in a formal definition but within a discussion on vegetation succession, climax and 'quasi-organism':

\section{The Ecosystem}

I have already given my reasons for rejecting the terms "complex organism" and "biotic community". Clements' earlier term "biome" for the whole complex of organisms inhabiting a given region is unobjectionable, and for some purpose convenient. But the more fundamental conception is, at is seems to me, the whole system (in the sense of physics), including not only the organism-complex, but also the whole complex of physical factors forming what we call the environment of the biome- the habitat factors in the widest sense. Though the organisms may claim our primary interest, when we are trying to think fundamentally we cannot separate them from their special environment, with which they form one physical system.

It is the systems so formed which, from the point of view of the ecologist, are the basic units of nature on the face of the earth.
This definition is simple and rich:

- First, it states that the ecosystem is both a physical system and a biological system. At the time, there was a strong debate about 'organismic' organization levels above the individual, assuming a purely biologically determined development of vegetation on large spatial scales. Tansley's purpose was to bring the physical world back within the scope of ecology, which in subsequent years has indeed been the case (Carpenter and Turner 1998).

- Second, Tansley does not explicitly refer to space or time in his definition. This should not be surprising any more than that the definition of the concept of an 'organism' need not refer to space or time. This does not mean that space or time is not important to the ecosystem (or to organisms): considering ecological processes implicitly involves considering time; and in field ecology, sampling requires specifying a spatial domain. Instead of space and time being terms within the definition, they should rather be considered 'nuisance parameters'. The absence of an explicit reference to space or time makes Tansley's ecosystem scaleindependent, as he showed himself by listing example ecosystems spanning orders of magnitude in size from the atom to the solar system (p. 300 of his article). This property distinguishes the ecosystem from the holocoen (Friederichs 1927) and may be responsible for its greater success (Figure 1).

- Third, the ecosystem is above all an intellectual construct. Tansley is clear about this later in the article where he writes (p. 300 of his article):

The whole method of science, as H. Levy ('32) has most convincingly pointed out, is to isolate systems mentally for the purposes of study, so that the series of isolates we make become the actual objects of our study. [...]. The isolation is partly artificial, but is the only possible way in which we can proceed. [footnote: The mental isolates we make are by no means all coincident with physical systems, though many of them are, and the ecosystem among them].

This remark is fundamental. It claims that the ecosystem is an abstraction developed for a particular purpose. There is no objective ecosystem because the choice of the investigator is central in its definition. 
- Fourth, it identifies the ecosystem as the basic brick of ecology. Ecology consists in seeing everything as ecosystems. Depending on what we mean by an 'organism-complex' or a 'physical complex', the definition can indeed encompass almost anything.

For Tansley, rather than an object, the ecosystem is a way to look at nature, a 'mental isolate' where physics and biology are the principle intellectual tools.

To build an ontology suitable for ecosystem modelling and theoretical development, we need to re-discuss these points in view of developments in ecology since 1935.

\section{Biology and Physics: Components or Aspects of the Eco- system?}

Tansley's words are ambiguous: the ecosystem associates an 'organism complex' with a 'complex of physical factors'. This can be interpreted in two ways: either the ecosystem is made of objects which belong to one of two classes, 'physical factors' or 'organisms', or the ecosystem is both a physical and a biological system, that is, is a dual object by nature.

Both interpretations are useful: the first can serve to classify ecosystems on a scale of increasing weight of the biological component, where for example, deserts are one end of the spectrum and tropical forests or coral reefs the other; following the second interpretation, one can choose to study the biotic community in a desert or the carbon fluxes of a tropical forest.

Tansley probably had in mind the first interpretation, very close to the old concept of biocenosis (Möbius 1877), because he further calls the 'complex of physical factors' the habitat. 'Habitat' is a particularly ambiguous word, which, according to authors, refers to the surrounding physical factors (for example, climatic and edaphic conditions), to a place (for example, 'benthic', 'pelagic'), or to a community of organisms (for example, a forest). Rather than proposing yet another definition of habitat, for the purpose of our ontology, we will name the place where organisms live and interact with each other the arena. An arena is a place where things happen and where observers (ecologists) may watch them happening. It contains organisms and organisms within the arena are close enough to each other to establish networks of interactions.

The arena has three important properties:

- It is the place of the world under focus, the place which makes sense for the ecological processes under study. Other things may happen outside the arena, but we are (temporarily) not interested by them.

- It is a physical container system: as a container of organisms it matches the classical definition of the habitat as a physical environment. Tansley's 'complex of physical factors' should be attached to the arena as a set of state variables describing its properties. Climatic variables and ecological niche axes are classic examples.

- It is the stage where organisms interact: within the arena, organisms know each other and can establish relationships. As such, the arena matches the definition of the habitat as a purely biotic environment made of interactions with other organisms.

There is no need for the arena to be spatially explicit, but it certainly contains properties linked to space: by construct, we assume that organisms inside the arena may be close enough to each other to interact, that is, a proximity relation is attached to the arena. The simplest arena assumes that all organisms within interact with every other, with possible restrictions based on organism roles (that is, hosts vs. parasites, preys vs. predators, competitors...). A more elaborate view would identify a proximity relation which would enable one to decide that some organisms are close enough to interact whereas others are not. Finally, the arena could be described as a fully spatially explicit place where interactions between organisms depend on their location within the arena or on euclidian distance between each other. As we see, there is some freedom in the degree of spatial description attached to an arena, depending on the purpose of the study.

In our view, the arena concept actually captures within a single word Tansley's 'complex of physical factors' and the ambiguous habitat, either as a physical environment, as a set of interactions, or even as a place. Depending on whether the emphasis is put on the container (the arena) or the content (the organisms), the ecosystem could be qualified as more abiotically driven or biotically driven, matching the above mentioned definitions of the habitat. From our definition, an ecosystem always has an arena by construct. In community or population ecology, the arena is implicit and the relations of organisms to the arena are simply ignored as not being the focus of study, or summarized as habitat quality or carrying capacity. Using this vocabulary, ecosystem engineers (Jones and others 1994) become organisms modifying their arena. 
It seems over time the second interpretation, the physical view, has come to dominate: Likens (1992) considers a gradient in focus on physics or biology when studying ecosystems. 'Focus' is understood there as a tendency for ecologists to study the dual object called ecosystem with the methods of physics, mainly thermodynamics, or with the tools of biology. In this interpretation, physics and biology become aspects of the ecosystem, rather than components. This is very powerful, because it allows us to adapt the ecosystem view to the problem under consideration. For a trophic network analysis, all organisms of an ecosystem can be viewed as energy sources, and their interactions as energy fluxes, the arena defining the total energy input. For an evolutionary perspective, the same ecosystem is viewed as a set of competing populations imposing selective pressures on each other, the arena defining the general constraints all have to obey. Using this interpretation, community and population ecology are purely biological approaches of the ecosystem, whereas biogeochemistry is a purely chemical/physical approach of the ecosystem. Analyzing an ecosystem simultaneously as a physical and biological system is difficult, but has proven fruitful in ecological stoichiometry (Elser and Hassett 1994) and biodiversity and ecosystem function studies (Naeem and others 1994; Loreau 2010). More recently, Loreau (2004) has shown that predator-prey relationships were affected by mass balance constraints.

Should we keep these interpretations separate or try to synthesize them? A possible reconciliation is suggested by Tansley himself: at the end of his definition, he states that organisms and their physical environment 'form one physical system', that is, the whole ecosystem itself can be seen as a physical system. This implies that organisms can be considered as physical systems themselves: it is true that all living organisms have to obey the laws of physics and can thus be considered as, for example, nodes in an energy flux network or structures influencing wind speed and climate. Following this view, all ecosystem components, the arena and the organisms, are physical systems, which means the whole system is easily studied with the tools of physics. Ecosystem science clearly followed this path (Carpenter and Turner 1998). An organism is just a specialized physical system which has additional properties making it different, like—at least—a definite lifespan and the ability to reproduce, and here biology and all its methods can come in. Considering organisms as physical systems with additional properties is the key to reconcile the component/aspect interpretation of the physics-biology duality.

\section{The Ecosystem as a Scale-Independent Concept}

Although there is no explicit reference to space (geometric, euclidian) or time in Tansley's definition of the ecosystem, one could argue that a physical system does imply some relation to space (Carnot 1824):

\begin{abstract}
A thermodynamic system is that part of the universe that is under consideration. A real or imaginary boundary separates the system from the rest of the universe, which is referred to as the environment or surroundings. ${ }^{1}$
\end{abstract}

There is some ambiguity in the role of space in this definition. The system itself does not refer to space, but one needs space to isolate a system for 'consideration': one has to define a boundary, a spatial concept. The thermodynamic system is independent from space, but a particular thermodynamic system must have been isolated from the rest of the universe by defining its boundary. In computer science, this distinction is identified by the terms class and instance (Shlaer and Mellor 1988). A class is a blueprint to create objects of a particular type, hence sharing common properties, called instances of this class. The thermodynamic system or the ecosystem definitions define a class of objects, but when used in a particular context these objects have to be instantiated-and this requires a spatial operation, defining a boundary. The role of space in the ecosystem class-instance relation is analogous to the role of computer memory allocation in a computer programming language classinstance relation: it is a technical operation required to make an instance that has nothing to do with the class properties themselves. Space as an explicit ecosystem property is optional, whereas acting on space is always necessary in the background to instantiate an ecosystem. We think that most of the problems and discussions about the role of space in ecosystems arise from the failure to identify these two roles of space in ecosystem ecology. Following this, the class definition (Tansley's definition) does not require space as a necessary component of ecosystems.

Space is needed when instantiating a particular ecosystem in a particular context, but no method is proposed in the definition. This has been a major problem for field ecologists: to be useful, concepts

\footnotetext{
1 As phrased by Wikipedia: http://en.wikipedia.org/wiki/ Thermodynamic_system.
} 
have to be applicable (Jax 2006). Some authors proposed making the ecosystem spatially explicit (Likens 1992), presumably to solve this problem. Lepczyk and others (2008) have proposed the term 'landscape' be applied to represent an association of space with an ecosystem. We will discuss later a possible alternative solution.

Similarly, there is no explicit reference to time in the ecosystem definition. Although considering the dynamics of ecosystems is clearly a mainstream of activity in current ecology, describing ecosystem structure statically has been considered a legitimate stream of ecology in the past (for example, Grubb and others 1963; Menaut and César 1979). The lack of reference to any time or space gives the ecosystem concept the highly prized property of being scale-independent (Allen and Hoekstra 1992).

To exclude a specific space and time in the class level definition of the ecosystem allows us to consider anything from the whole biosphere to a bacteria cell as an ecosystem and makes it possible to use it as 'the basic bricks of ecology'. For example, most landscape ecologists consider the landscape as a relatively large object. We do not know of any examples of studies dealing with a landscape of a few square millimeters, as would be possible in soil ecology, for example. Usage rather than logic restrains the application of the landscape concept to all the spatial scales to which it could potentially apply: people tend to consider that the 'ecosystem scale' is somewhat smaller than the 'landscape scale', landscape being used in its common meaning of a large area of land. In fact, the landscape in its modern meaning could be interpreted as the 'spatially explicit ecosystem' of Likens (1992).

\section{The Ecosystem as an Artificial Construct}

For Tansley, an ecosystem is a mental isolate or a system we isolate for the purpose of the study. This differs from the similar concept of the holocoen (Friederichs 1927), which was defined as 'a naturally delimited part of the biosphere' (Jax 2006). This concept is now unknown apart from science historians whereas the ecosystem concept has spread widely (Figure 1) and is well-known outside

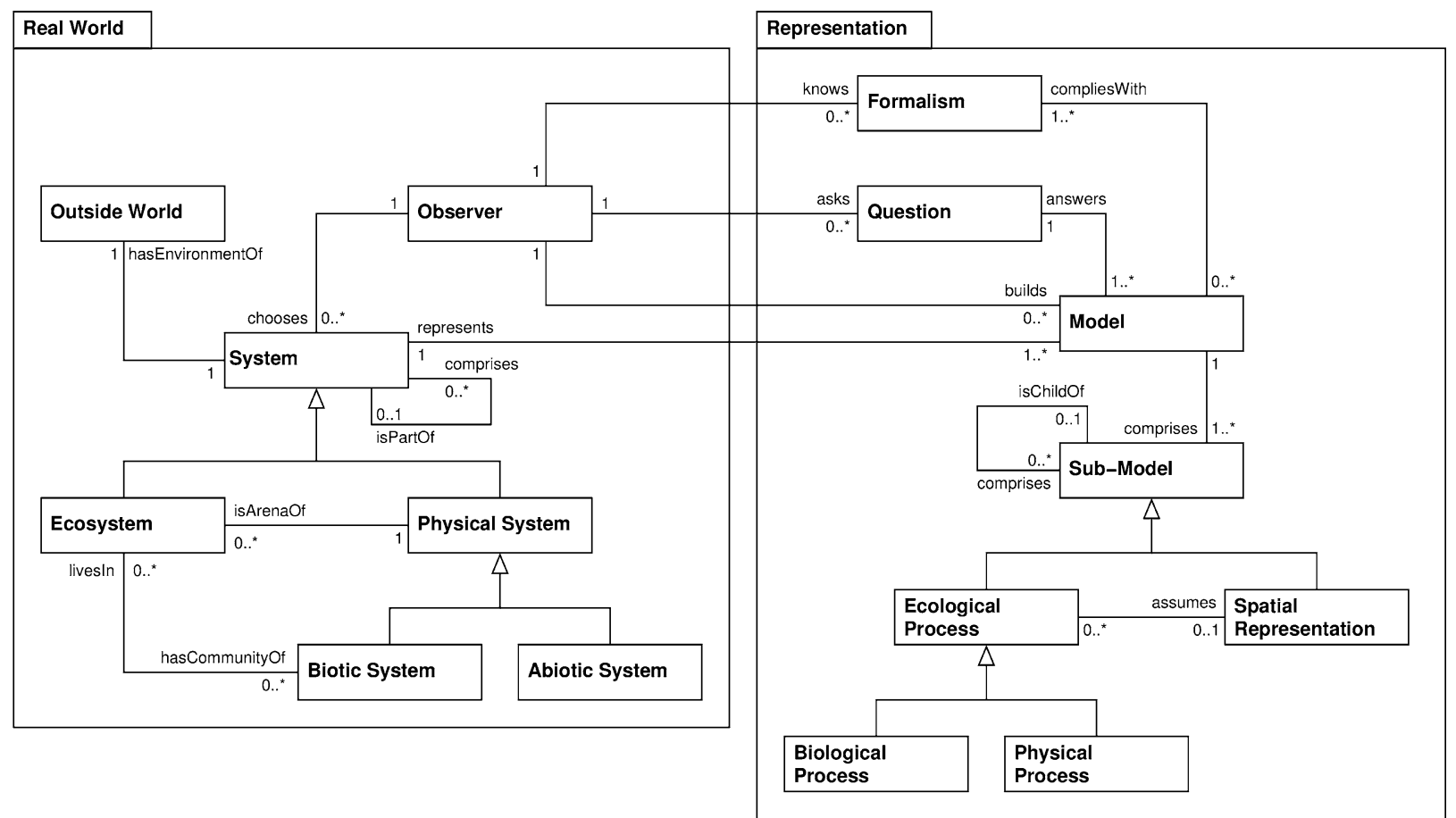

Figure 2. UML class diagram describing the relations between the ecosystem as a particular system, the outside world, organisms generalized here to living systems, and physical systems. UML (http://www.uml.org/) notations: boxes are generic classes of objects; lines represent associations between classes, qualified by phrases and multiplicities; the triangular arrow is the specialization or 'is a' relation, for example, a physical system is a specialized version of a system and a biological system is a specialized version of a physical system. The diagram is best read by forming sentences from class names, association phrases and multiplicities. For example, 'a physical system may be the arena of many ecosystems'; 'an ecosystem has a community of biotic systems'. See Table 1 for definitions of classes and Appendix S4 for a brief description of the UML language. 
ecology. Making a 'mental isolate' is a particular operation requiring an observer: contrary to the holocoen, the ecosystem does not exist without an observer to define it. Notice that this operation is different from modelling: a model is a representation of the real world, whereas an ecosystem-and even, should we say, any system in the sense of physics (Carnot 1824) -is a part of the real world that an observer decides to isolate (Figure 2). This isolation may or not be physically possible: for example, the rhizosphere ecosystem is a mental isolate of the real world that is impossible to physically sample in the field.

From the scale independence property, the observer may define an ecosystem at any scale, as long as it satisfies the biology/physics duality. It follows that it is possible to define nested ecosystems, that is, ecosystems within ecosystems. We can then derive from Tansley's definition that the ecosystem is potentially a recursive, self-similar object, as already recognized by Pickett and Cadenasso (2002). By self-similar we mean in terms of its definition, not that it need contain the same parts.

The problem is that the 'nesting' operator for ecosystems is not based on a single commonly agreed upon relation, but on many. We identified the following methods for nesting ecosystems (Figure 3)-there may be others:

1. Community sub-sampling: The community of organisms of an ecosystem is made of populations belonging to various classes according to taxonomic, functional or other classification; a straightforward method for defining a sub-ecosystem within another one is to restrict its community to a few of the initial groups. One can, for example, decide to confine a lake ecosystem by considering only phytoplankton within a larger 'full trophic web'.

2. Arena structure nesting: In an ecosystem's arena, we can often recognize different physical media separated by interfaces. These parts commonly host different subsets of organisms because the physical processes inside and among them differ. Ecologists use this compartmentalization to define sub-ecosystems within larger ones: for example, the soil ecosystem within a forest or the benthos in a lake.

3. Spatial zoom nesting: If an ecosystem has been isolated as a landscape, then a smaller area within the first one contains an ecosystem that may be nested in the first one. There is uncertainty here, because the zoomed-in ecosystem may or may not be the same as the first. Remember that the ecosystem is scale-independent: there is no guarantee that a smaller spatial domain contains fewer components of the ecosystem, it may be homeomeric, even if classical relations such as the species-area curve suggest otherwise.

4. Temporal nesting: Similarly to the previous method, and with the same restrictions, we can

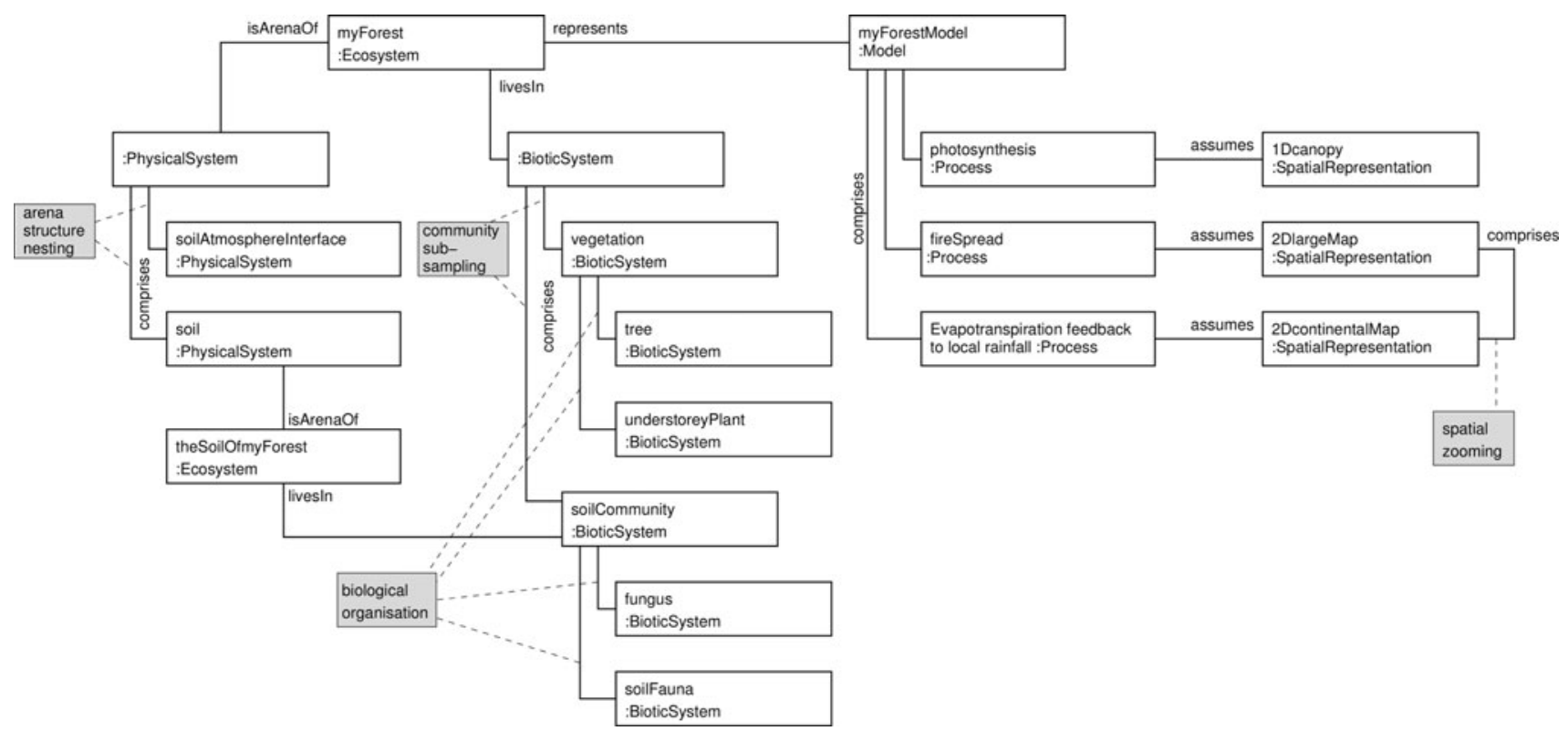

Figure 3. A forest ecosystem using the ontology, illustrating the common nesting methods for ecosystems. UML object diagram: Boxes and relations on this diagram represent instances (= individual members of classes, for example, vegetation is an instance of a biotic system while soil community is another one) of the classes and relations of Figure 2. Grey boxes are annotations qualifying four of the ecosystem nesting operators. 
isolate potential sub-ecosystems by sampling a long lasting ecosystem on a shorter time period. A classic example is vegetation succession: successive vegetation stages can be identified as sub-ecosystems of the whole succession series, considered as a 'large' ecosystem.

5. Biological organization hierarchy nesting: A nested hierarchy of cells, tissues, organs, individuals, populations and communities exists in the real world (Allen and Hoekstra 1990; Aleshchenko and Bukvareva 1994; Lidicker 2008). These units are not based on spatial scale as clearly some individuals and organs are larger than some populations. In addition some populations are not wholly contained within a single community (Allen and Hoekstra 1992). There is a container/contained relation between successive levels. There is probably no single hierarchy, that is, hierarchies involving clonal plant and colonial animals would probably slightly differ from hierarchies of groups with clearly identified individuals; autonomous cells of unicellular organisms are lumping all the organization levels between the cell and the individual into a single 'individual cell'. The traditional scope of ecology usually tends to consider organization levels above the individual, that is, populations and communities, which makes this nesting method a particular case of the community sub-sampling method. However, this method is richer: we could define the ecosystem made of a single individual and its physical environment; and, if we generalized the ecosystem further down the biological hierarchy, by replacing Tansley's 'organism' with 'biotic system', we could also study cells within tissues or organs within individuals with the methods of ecology, possibly opening new areas of research for ecology.

To our knowledge, these five nesting relations have not been explicitly identified before, which may explain some of the confusion surrounding the hierarchical organization of ecological objects, where the concepts of level of organization, spatial and temporal scale are used inconsistently. Interestingly, there is little agreement at levels beyond the community (that is, at the 'ecosystem' level in the traditional view) (Lidicker 2008)-except maybe when using the landscape concept (Lepczyk and others 2008).

These nesting relations make the ecosystem an archetype of a complex system in the commonly accepted sense of 'system made of interacting components that are themselves systems, displaying emergent properties' (Jorgensen and others 1992; Müller 2004). Although emergent properties are not part of the ecosystem definition, they are expected to occur in ecosystems (Grimm and Railsback 2005). Interestingly, Tansley's intuition of observer-dependence recently appeared in modern definitions of emergence in complex systems (Dessalles and others 2007). The ecosystem is, definitely, a subset of the real world as a self-similar complex system, used by field ecologists as a perceptive filter to restrain the amount of information to be gathered, and by theory and model developers to make the identification and quantification of key driving processes possible.

\section{Everything is an Ecosystem}

Ecology consists in viewing everything as ecosystems. If ecosystems are scale-independent, recursive, biological and physical systems, defined by an observer for some purpose, then almost any object of the real world can be studied as an ecosystem.

Systems involving humans, including cities and societies can clearly be studied as ecosystems with the tools of ecology. Extending Tansley's definition by replacing 'organism' with 'biotic system', it becomes possible to study, for example, cells within a tissue or organelles within a cell as ecosystems with the methods of ecology. Ecologists dealing with protists and unicellular plankton are used to considering cells within their physical environment, why could not liver cells within an animal be studied in the same way? There is a cultural gap between ecologists and cell biologists, which probably only relies on the ecosystem definition being restricted to organisms. Rather than a treason to Tansley's idea, we consider this an expansion of its scope allowing other systems to be explored as ecosystems. If we generalize even further and consider replacing biotic systems with 'objects which have a finite lifespan, can maintain and reproduce themselves, and interact with their local environment', then the ecosystem definition can apply to systems outside the traditional scope of ecology, like for example, virtual communities of agents in silico (multi-agent systems: Ferber 1995), or communities of economic agents (for example, industrial ecology: Frosch and Gallopoulos 1989).

We take the great success of the concept (Figure 1) as a testimony of its quality and interest for developing a scientific approach of the living world. Tansley's definition is a brilliant intellectual construct which nevertheless has posed some problems in its practical application which we now discuss. 


\section{The Ecosystem in Practice}

Ecologists face three problems associated with the ecosystem definition:

- The duality problem: How can you study a system defined as both physical and biological?

- The boundary problem: How, in practice do we sample ecosystems in the field? Can we delineate them?

- The abstraction problem: To what level of detail should an ecosystem be modelled?

\section{The Duality Problem}

As early as Lindeman (1942), the duality between physics and biology was identified as posing methodological problems because of contradictions in the objectives and methods of physics and biology:

- Branches of physics of interest to ecology (thermodynamics and system dynamics) aim to understand and predict the fluxes of matter and energy, the maintenance of structures in time and their spread in space. The underlying law is that of energy and mass conservation. Thermodynamic systems are described by state variables that exhibit continuous variations over time. Much of this work uses systems of differential equations (SDEs) and associated mathematical techniques.

- Branches of biology of interest to ecology aim to understand and predict the fate of large scale, integrated systems (populations and communities) from the properties and interactions among their components (individuals, sub-populations, but also organs or cells). The underlying laws are those of genetics, behaviour, demography and evolution. The associated modelling tools are of two main types: SDEs and other similar mathematical tools (for example, population dynamics models: Caswell 1989) and individual-based models (IBMs) relying on computer simulation (for example, Grimm and Railsback 2005).

Ecologists are faced with a representation problem: ecosystems appear as dual objects, obeying both biological and physical laws, just as light obeys both wave and particle physics. Methodological issues arise from this duality:

1. the state variables/differential equations methods of physics and theoretical population biology are largely incompatible with computer simulation methods, although some pioneering works attempt to develop analytical tools
(Gratzer and others 2004) for taking account of emergence (a 'biological' property) in non-IBMs based on results from IBMs;

2. traditional systems of equations quickly become intractable for even the simplest ecosystems (for example, Loreau 2004);

3. strong conclusions on model behaviour (for example, stability, equilibrium) cannot be obtained from IBMs which rely on simulation to analyze their long-term behaviour.

The methodological gap between SDEs and IBMs is still wide. Intuitively, we may use SDEs within an IBM to couple physics and biology. However, a biological system has a finite lifetime-it is born and dies. Although it lives it processes matter and energy as any other physical system. Hence if a system of equations applying to state variables must be used to model some physical processes within a biological system, what happens in an IBM when biological objects are created and destroyed based on their interactions?

Villa (2001) calls the problem we are facing here a 'multi-paradigm' problem: ecology as a synthetic science is based on different representations that require different tools; he proposes a modelling framework to deal with this issue. To us, the key difficulty of ecological modelling is to reconcile the classical state-variable, physics based, approach, and the IBM, biology based, approach because ecosystems are both physical and 'biological-social' systems. IBMs can represent emergence, but we need to be able to summarize their behaviour in elegant mathematical formulation such as those of physics. Conceptual frameworks exist in computer science to do this: the integrated modelling architecture (Villa 2001), the aspect-oriented thinking (Flint 2006), and in some ways the DEVS formalism (Zeigler and others 2000) (Appendix S2).

\section{Space and the Boundary Problem}

In practice, we need a way to define a boundary to instantiate a particular ecosystem. In the field, this means practical criteria to decide whether a given area or set of organisms must be sampled or not. This problem has long been identified: Jax and others (1998) proposed two methods for identifying 'ecological units' in the field: using spatial boundaries or functional boundaries. To us, the first method is dealing with landscapes (Jax 2006) whereas the second deals with ecosystems. The biggest problem when using the landscape concept and its purely arbitrary spatial boundaries is that there is no guarantee that the resulting ecosystem is relevant to the problem under study. 
Another method is possible. Because an ecosystem is instantiated for a particular purpose, the representation of the real world used in a particular instance will only focus on a tractable number of ecological processes. By ecological processes, we mean interactions among ecosystem components that can be explained using causal relations'. These processes will possibly have properties which 'naturally', 'logically' or 'computationally' suggest a particular spatial domain and representation: for example, although mortality need not refer to space, seed dispersal implies by construct a relation to a geometric space. This may be clearer with an example (Figure 4):

A lake is a clearly delineated ecosystem: its boundaries are easy to agree on, and as a result a whole branch of ecology (limnology) uses these systems as their basic ecosystem unit. There is a rich literature on the study of pelagic and benthic trophic networks within lakes. But many aquatic systems are actually heterotrophic, and depend on external inputs of terrestrial organic matter from the surrounding catchment (del Giorgio and others 1997). One may thus question whether isolating the lake from its surrounding water catchment is a good idea. To correctly model or measure the carbon fluxes associated with the 'microbial loop', one may need to consider the lake within its catchment as the relevant spatial unit. In addition, if one wants to model/measure the effect of migratory birds feeding on fish, it may make sense to consider the lake as a point on a migratory route. In this case, according to the detail we want to include in our representation of the trophic network, we may select three different spatial domains for the same ecosystem.

In other words, the need to make the representation of an ecosystem spatially explicit together with the relevant spatial representation depends upon the context of the study, which results in the selection of a set of ecological processes that need to be represented and/or measured. As in our lake example, the relevant spatial representations are associated with ecological processes, not with the ecosystem. It follows that the ecosystem cannot have only one associated spatial scale, but as many as the considered ecological processes require. There is no reason for an ecosystem to have a single spatial domain or extent, it can have none or many. Contrary to the landscape, the ecosystem is not spatially consistent, that is, it does not have a single boundary, but has many, possibly overlapping, boundaries, as required by the functional processes under consideration (Figure 2). The same holds for

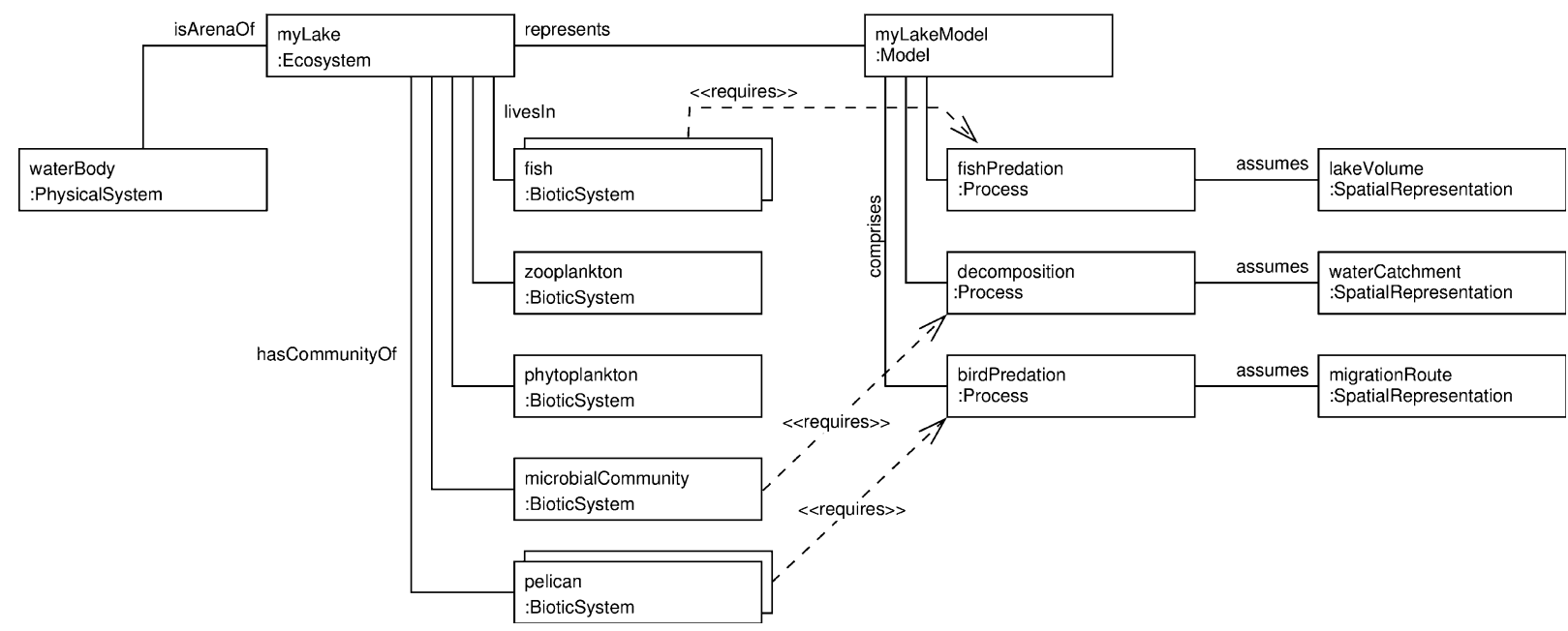

Figure 4. A lake ecosystem represented using the ontology, illustrating the multiple spatial representations associated to an ecosystem. UML object diagram: Boxes and relations on this diagram represent instances (= individual members of classes, for example, zooplankton is an instance of a biotic system while phytoplankton is another one) of the classes and relations of Figure 2. In this example, three spatial models (and domains), each associated to a particular ecological process, are used to represent the same ecosystem. The selection of the processes considered depends on user choice, motivated by logical constraints such as including pelicans requires consideration of predation by birds, which requires modelling the lake as a point on a migration route because of the behaviour of these birds'. 
time representations, because they too will be associated with processes rather than the whole system.

This view has the advantage of clarifying the relation of ecosystems to space (and time): it is a complex relation because it is a compound, context-dependent, relation. Depending on observer choice, some ecological processes are selected, which imply some spatial representations. There is no reason for these representations to be identical, so the ecosystem ends up as a mixed-scale object, which may explain part of the discussion on the ecosystem definition and its relations to space (for example, Likens 1992; Allen and Hoekstra 1992). This also explains why the holocoen is not a practical concept: in such an object, one must consider an infinity of ecological processes, hence an infinity of overlapping spatial domains, which is intractable. The 'mental isolate' we must inevitably impose is crucial, because it allows us to only consider a finite, limited and tractable number of ecological processes and their associated spatial representations.

This implies two broad approaches to field ecology. The first selects a particular spatial domain and then looks at the possible ecosystems resulting from this sampling. The second uses the ecosystem concept to select particular processes and then adapts the sampling to the different spatial domains relevant for these processes. There is no general method for deducing a relevant spatial domain or sampling window from an ecological process, but on this basis a formal argument for a sampling strategy can be proposed. The former approach is more adapted to exploratory studies where many of the interactions within the studied system are unknown, whereas the latter is more adapted to analytic studies of systems where the dominant processes have already been identified.

\section{The Abstraction Problem}

In artificial intelligence, Tansley's 'mental isolate' is viewed as an 'abstraction' (Zucker 2003). Abstraction is the process by which we focus on some aspects of the world to the exclusion of others. This takes place in two contexts: selecting an ecosystem in the real world and constructing an ecosystem model by selecting relevant ecological processes (Figure 2). Both operations are subjective as to what to include and the level of detail to represent or sample. This is common practice in ecology, and there is no objective way to prescribe this: within the same study context, different levels of detail are possible.
The abstraction problem arises when the choice to elaborate parts of an ecosystem in greater or lesser detail affects the outcomes of the study. Ideally, models representing the same ecosystem using different levels of detail (or abstraction level) should yield the same outcomes. It seems a good property that predictions on ecosystem fate are independent of the abstraction level.

Unfortunately, the abstraction problem regularly arises in the literature, sometimes feeding intense debate. Hulot and others (2000) demonstrated that describing a trophic network as a chain or as a network completely changed the response of the ecosystem to nutrient enrichment. In a more formal test, Lazzaro and others (2009) demonstrated that a trophic network described in detail had different network-level properties (for example, connectance, number of top predators, and so on) from a trophic network described using lumped species. Pacala and Deutschman (1995) and Simioni and others (2003) demonstrated that the formerly ignored or empirically averaged spatial structure of ecosystems had significant effects on primary production. Finally, the debate on biodiversity and ecosystem functioning (Hector and others 1999; Loreau and others 2001) centred around re-including diversity as a key component to explain major ecosystem properties.

There is very little hope of solving this problem: the complexity of natural systems, and of ecosystems as subsets of them, almost certainly precludes the existence of a single, ideal representation. Whereas it may be possible to model them, it is practically impossible to sample all populations within a community at the same abstraction level, either because the total list of species to consider in an ecosystem is unknown, or because individual organisms span orders of magnitude in size. For example, almost all trophic network studies involve a remarkably undetailed 'decomposer' box contrasting with the sometimes very detailed description of the other compartments; vegetation studies often detail the tree community and lump the soil as a single component. In most cases, the consequences of doing so on whole system level properties are unknown.

Many models can represent the same ecosystem, all bearing a part of truth, some being more efficient for some particular purpose, and it is naive to look for a single, 'best' representation. However, there are possible ways of improving the situation, by:

1. Reducing empiricism in model construction: Choosing an abstraction level is rarely a delib- 
erate act. It is most often based on common uses and practices rather than well-grounded methodology. Being able to assess and compare the abstraction level of different models could assist, using for example the ODD standard protocol for describing and publishing ecological models (Grimm and others 2006; Grimm and others 2010).

2. Explicitly designing scaling up methods on ad hoc experiments rather than on empiricism: Scaling methods have been proposed to change the abstraction level without losing the relevant details, that is, maintaining properties of interest in the more detailed approaches (Grimm and others 1996; Smith and others 2001; Barnes and Roderick 2004; Boulain and others 2007, 2009). These approaches have been formalized in theories of representation changes (Giunchiglia and Walsh 1992) or as extension of existing formalisms (Giambiasi and Carmona 2006).

3. Designing modelling tools that enable nonexperienced ecological modellers to examine the consequences of the choice of an abstraction level: Individual-based ecology (Grimm and Railsback 2005) and agent-based simulation (Ferber 1995; North and Macal 2007) give a framework in which to develop simulation models where abstraction level can be made explicit and controlled, a first step to reduce empiricism in model building. Multi-agent simulation (MAS) software exist (for example, SWARM: Minar and others 1996; NetLogo: Wilensky 1999; Repast: North and others 2007; GAMA: Amouroux and others 2009), largely based on social science concepts-ecology still lacks a dedicated MAS software.

We advocate that two types of studies, (1) finding and/or standardizing methods for scaling up and

(2) comparing representations of the same system at different levels of abstraction, are of key importance for ecosystem science. They seem the only options to limit empiricism in model construction, a prerequisite to answer the abstraction problem.

\section{An Ontology for Ecosystems}

The ontology we propose here aims at expressing the richness of the ecosystem definition in a formal way. We hope that this will facilitate the manipulation of the ecosystem concepts, both in modelling and experimental or field studies. The ontology has been written using the Protegé Software (Gennari and others 2003: http://protege.stanford.edu/) and can be downloaded from http://threeworlds. biologie.ens.fr/ or as Supplementary material with this article.

The ontology concepts have been defined within two domains, the real-world domain and the representation domain (Table 1). In selecting the properties required in formal definitions, we have been careful to include only properties that can be used in practice to decide to which class an object belongs. Readers should be aware that what is excluded from the definitions is at least as important as what has been included, and that relations between concepts are as important as the concepts themselves. Appendix S3 contains a fuller discussion of each definition. We illustrate the relations between the concepts of our ontology using the unified modelling language (UML: http://www. uml.org/; Mellor and Balcer (2002); Silva Parreiras and Staab (2010); Figure 2 and Appendix S4).

\section{Discussion}

Our aim has been a modern interpretation of the original ecosystem definition (Tansley 1935) that may serve as a conceptual framework for discussion and for ecological modelling. We have presented the concept as an ontology for ecosystems, adding little to Tansley's concept while keeping the definitions simple (Table 1; Figure 2).

Various authors have introduced new ideas to the ecosystem concept to maintain contemporary relevance. At times this has specified properties an ecosystem must exhibit that has complicated the issue to the point that $\mathrm{O}^{\prime}$ Neill (2001) has suggested abandoning the concept. We argue that the ecosystem concept as it was first proposed is sufficiently rich as not to require additional terms but nevertheless still yields an elaborate ontology. Our focus has been to refrain from over-specifying the definition, an approach we believe, likely to restrict the possible applications of the concept and lead to a loss of generality.

Our recursive ecosystem matches the definition of the holons of the hierarchy theory $\left(\mathrm{O}^{\prime} \mathrm{Neill}\right.$ and others 1986): holons are simultaneously components of a system and compound objects, themselves systems of holons. Hierarchy theory attempts to delineate its hierarchical levels and holons based on the relation between phenomena and spatiotemporal scales (Ratzé and others 2007), assumed to be identifiable from the existence of scale-related discontinuities and thresholds in state variable changes. Rather than a founding principle of the hierarchy theory, this should be considered as a hypothesis, actually a very interesting one: we 
Table 1. Ontology Definitions

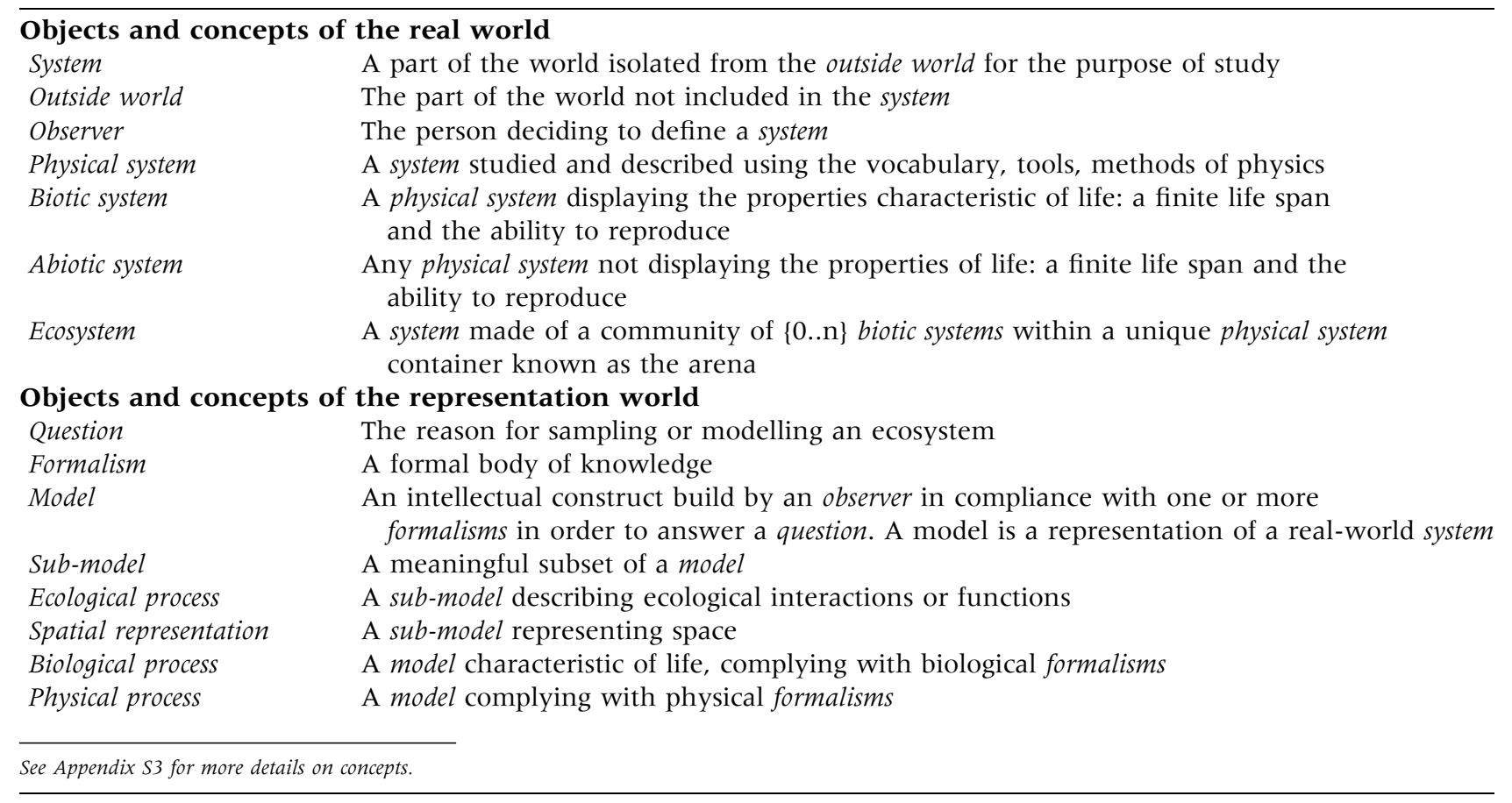

would have made a huge step forward if we could consider that the relations between scales and hierarchies proposed by this theory were proven true. Not including holons and hierarchy theory in the ecosystem concept provides the necessary freedom for people to experiment with the concept and test whether holons or ecosystems really relate to spatio-temporal scales in the way proposed by the hierarchy theory. As said by Ratzé and others (2007): 'one of the greatest challenges for mechanistic ecological modelling is to meaningfully connect the levels of organization.' Meaning cannot be prescribed, one has to test and experiment to prove an idea makes sense to a problem.

The use of objective criteria to identify holons within a hierarchy conflicts with Tansley's intuition that the ecosystem is an ad hoc subset of the real world. The only justification for identifying objects in an ecosystem is whether they make sense for the study-it is a practical approach. After all, even a concept apparently as objective as the individual can be debated: in clonal plants, spatial and genetic individuals do not match (Hartnett and Bazzaz 1985), and it is almost impossible to delineate individuals in fungi. Peer agreement on internal consistency, rather than objectivity, is the ultimate criterion for proposing a particular ecosystem description. As there is no reference to an absolute object, we can accept the coexistence of different ecosystems for the same real-world object, and use appropriate techniques (Flint 2006) to make their representations compatible in a formal computing framework.

The inclusion of complexity in any definition of ecosystem is problematic not only because there is no general agreement on the way to measure complexity in the real world (Anand and Orloci 2000; Parrott 2005; Green and Sadedin 2005) but also because the first theorem applying to Kolmogorov's algorithmic complexity proves that it is not computable! Although structure or organization (Anand and Orloci 2000; Zhang and Wu 2002) and diversity (Ricotta 2000) are often recognized as key features of complex systems and seem commonly observed features of ecosystems, we believe it should not be included as a definition item.

Emergence is commonly recognized as an important attribute of complex systems (see Reuter and others (2005) for refined definitions of emergence). Using network or graph representations of complex systems, various ways to assess and measure emergence have been proposed: control theory (Kalman 1963, quoted by Fath 2004), small world property (Watts and Strogatz 1998), and more recently algorithmic complexity associated with a descriptive language within a hierarchical context (Dessalles and others 2007). In classical ecology, broad ecosystem properties such as stability, resilience, and also services to human societies, are supposed to emerge from interactions 
between ecosystem components (Loreau 2000). Nevertheless, it is recursivity alone that makes the ecosystem potentially complex. Although we may expect ecosystems to display emergence and complex behaviour, Tansley's definition is mute on this point and usefully so. Ecosystem models can be constructed to exhibit emergent properties or not as the case may be. Rather than setting the ecosystem in a too tight frame, the main value of complex system theory for ecosystem science is in addressing the abstraction problem. Thinking of the ecosystem as a complex system allows us to understand that although there is no unique representation of an ecosystem, all must yield the same predictions in the end. In a good model, prediction must be independent of the abstraction level. Although ecosystems are simplifications of a complex world, they seem themselves to exhibit sufficient complexity as to prevent this property holding.

\section{Conclusion}

Tansley's definition of the ecosystem has experienced considerable success but problems still remain. The duality and boundary problems can be solved using modern computer science techniques and a clear restatement of the concepts used in the ecosystem definition. The abstraction problem can only be improved by empirical comparisons of models using different abstraction levels, which in turn requires standardized modelling practices based on commonly understood terms. The key properties of the ecosystem being multi-disciplinary, recursive, scale-independent and observerdependent allow the proposal of a definition compatible with the recent theories of hierarchy and complex systems, which serve as a basis for most modern simulation techniques in computer science. We demonstrate that, by going back to Tansley's words, we can propose an ontology based on simple definitions, that could serve as a basis for modelling ecosystems. It is our hope that this ontology be discussed, refined and populated with examples that can then be used to define forthcoming tools and modelling software.

\section{ACKNOWLEDGMENTS}

We thank Gérard Lacroix, Hauke Reuter and an anonymous referee for challenging and constructive comments on a previous version of this article. This work has been funded by the French Agence Nationale de la Recherche (grant ANR-07-CIS7001, 'the 3Worlds project').

\section{REFERENCES}

Aleshchenko GM, Bukvareva EN. 1994. A variant of combining diversity models in biosystems of population and biocoenotic levels. Zh Obsh Biol 55:70-7.

Allen TFH, Hoekstra TW. 1990. The confusion between scaledefined levels and conventional levels of organization in ecology. J Veg Sci 1:5-12.

Allen TFH, Hoekstra TW. 1992. Towards a unified ecology. New York: Columbia University Press.

Amouroux E, Chu TQ, Boucher A, Drogoul A. 2009. GAMA: an environment for implementing and running spatially explicit multi-agent simulations. Agent Comput Multi-Agent Syst 5044:359-71.

Anand M, Orloci L. 2000. On hierarchical partitioning of an ecological complexity function. Ecol Model 132:51-62.

Barnes B, Roderick ML. 2004. An ecological framework linking scales across space and time based on self-thinning. Theor Popul Biol 66:113-28.

Boulain N, Simioni G, Gignoux J. 2007. Changing scale in ecological modelling: a bottom up approach with an individual based vegetation model. Ecol Model 203:257-69.

Boulain N, Cappelaere B, Ramier D, Issoufou HBA, Halilou O, Seghieri J, Guillemin F, Oi M, Gignoux J, Timouk F. 2009. Towards an understanding of coupled physical and biological processes in the cultivated Sahel-2. Vegetation and carbon dynamics. J Hydrol 375:190-203.

Carnot S. 1824. Réflexions sur la puissance motrice du feu et sur les machines propres à développer cette puissance. Paris: Bachelier.

Carpenter SR, Turner MG. 1998. At last: a journal devoted to ecosystem science. Ecosystems 1:1-5.

Caswell H. 1989. Matrix population models. Sunderland: Sinauer.

De Nicola A, Missikoff M, Navigli R. 2009. A software engineering approach to ontology building. Inform Syst $34: 258-75$.

del Giorgio PA, Cole JJ, Cimbleris A. 1997. Respiration rates in bacteria exceed phytoplankton production in unproductive aquatic systems. Nature 385:148-51.

Dessalles JL, Müller JP, Phan D. 2007. Emergence in multi-agent systems: conceptual and methodological issues. In: Phan D, Amblard F, Eds. Agent-based modelling and simulation in the social and human sciences. Oxford: The Bardwell Press.

Elser JJ, Hassett RP. 1994. A stoichiometric analysis of the zooplankton-phytoplankton interaction in marine and freshwater ecosystems. Nature 370:211-13.

Fath BD. 2004. Distributed control in ecological networks. Ecol Model 179:235-45.

Ferber J. 1995. Les systèmes multi-agents. Vers une intelligence collective. InterEditions, Paris.

Flint SR. 2006. Aspect-oriented thinking-an approach to bridging the disciplinary divides. PhD thesis, Australian National University, Canberra.

Friederichs K. 1927. Grundsatzliches über die Lebenseinheiten hoherer Ordnung und den ökologischen Einheitsfaktor. Naturwissenschaften 15:153-7.

Frosch RA, Gallopoulos NE. 1989. Strategies for manufacturing. Sci Am 261:144-52.

Gennari JH, Musen MA, Fergerson RW, Grosso WE, Crubézy M, Eriksson H, Fridman Noy N, Tu SW. 2003. The evolution of 
Protégé: an environment for knowledge-based systems development. Int J Hum Comput Stud 58:89-123.

Giambiasi N, Carmona JC. 2006. Generalized discrete event abstraction of continuous systems: GDEVS formalism. Simul Model Pract Theory 14:47-70.

Giunchiglia F, Walsh T. 1992. A theory of abstraction. Artif Intell 56:323-90.

Gómez-Pérez A, Fernández-López M, Corcho O. 2004. Ontological engineering: with examples from the areas of knowledge management, e-commerce and the semantic web. NewYork: Springer.

Gratzer G, Canham C, Dieckmann U, Fischer A, Iwasa Y, Law R, Lexer MJ, Sandmann H, Spies TA, Splechtna BE, Szwagrzyk J. 2004. Spatio-temporal development of forests-current trends in field methods and models. Oikos 107:3-15.

Green DG, Sadedin S. 2005. Interactions matter-complexity in landscapes and ecosystems. Ecol Complex 2:117-30.

Grimm V, Railsback S. 2005. Individual-based modelling and ecology. Princeton: Princeton University Press.

Grimm V, Frank K, Jeltsch F, Brandl R, Uchmanski J, Wissel C. 1996. Pattern-oriented modelling in population ecology. Sci Total Environ 183:151-66.

Grimm V, Berger U, Bastiansen F, Eliassen S, Ginot V, Giske J, Goss Custard J, Grand T, Heinz S, Huse G, Huth A, Jepsen J, Jorgensen C, Mooij W, Muller B, Pe' er G, Piou C, Railsback S, Robbins A, Robbins M, Rossmanith E, Ruger N, Strand E, Souissi S, Stillman R, Vabo R, Visser U, DeAngelis D. 2006. A standard protocol for describing individual-based and agentbased models. Ecol Model 198:115-26.

Grimm V, Berger U, DeAngelis DL, Polhill JG, Giske J, Railsback SF. 2010. The ODD protocol: a review and first update. Ecol Model 221:2760-8.

Grubb PJ, Lloyd JR, Pennington TD, Whitmore TC. 1963. A comparison of montane and lowland rain-forest in Ecuador. 1. The forest structure, physiognomy, and floristics. J Ecol 51:567-601.

Gruber TL. 1993. A translation approach to portable ontology specifications. Knowl Acquis 5:199-220.

Guarino N. 1995. Formal ontology, conceptual analysis and knowledge representation. Int J Hum Comput Stud 43: 625-40.

Hartnett D, Bazzaz F. 1985. The regulation of leaf, ramet and genet densities in experimental populations of the rhyzomatous perennial Solidago canadensis. J Ecol 73:429-43.

Hector A, Schmid B, Beierkuhnlein C, Caldeira M, Diemer M, Dimitrakopoulos P, Finn J, Freitas H, Giller P, Good J, Harris R, Högberg P, Huss-Danell K, Joshi J, Jumpponen A, Körner C, Leadley P, Loreau M, Minns A, Mulder C, DG O, Otway S, Pereira J, Prinz A, Read D, Scherer-Lorenzen M, Schulze E, Siamantziouras A, Spehn E, Terry A, Troumbis A, Woodward F, Yachi S, Lawton J. 1999. Plant diversity and productivity experiments in European grasslands. Science 286:1123-7.

Hulot F, Lacroix G, Lescher-Moutoué F, Loreau M. 2000. Functional diversity governs ecosystem response to nutrient enrichment. Nature 405:340-4.

Jax K. 2005. Function and "functioning" in ecology: what does it mean? Oikos 111:641-8.

Jax K. 2006. Ecological units: definitions and application. Q Rev Biol 81:237-58.

Jax K. 2007. Can we define ecosystems? On the confusion between definition and description of ecological concepts. Acta Biotheor 55:341-55.
Jax K, Jones CG, Pickett STA. 1998. The self-identity of ecological units. Oikos 82:253-64.

Jones C, Lawton J, Shachak M. 1994. Organisms as ecosystem engineers. Oikos 69:373-86.

Jorgensen S, Patten B, Straskraba M. 1992. Ecosystems emerging: towards an ecology of complex systems in a complex future. Ecol Model 62:1-27.

Lazzaro X, Lacroix G, Gauzens B, Gignoux J, Legendre S. 2009. Predator foraging behaviour drives food-web topological structure. J Animal Ecol 78:1307-17.

Lepczyk CA, Lortie CJ, Anderson LJ. 2008. An ontology for landscapes. Ecol Complex 5:272-9.

Lidicker WZ. 2008. Levels of organization in biology: on the nature and nomenclature of ecology's fourth level. Biol Rev 83:71-8.

Likens GE. 1992. The ecosystem approach: its use and abuse. Oldendorf/Luhe: Ecology Institute.

Lindeman RL. 1942. The trophic-dynamic aspect of ecology. Ecology 23:399-417.

Loreau M. 2000. Biodiversity and ecosystem functioning: recent theoretical advances. Oikos 91:3-17.

Loreau M. 2004. Does functional redundancy exist? Oikos 104:606-11.

Loreau M. 2010. Linking biodiversity and ecosystems: towards a unifying ecological theory. Philos Trans $\mathrm{R}$ Soc Ser B 365:49-60.

Loreau M, Naeem S, Inchausti P, Bengtsson J, Grime JP, Hector A, Hooper DU, Huston MA, Raffaelli D, Schmid B, Tilman D, Wardle DA. 2001. Ecology-biodiversity and ecosystem functioning: current knowledge and future challenges. Science 294:804-8.

Mellor SJ, Balcer M. 2002. Executable UML: a foundation for model-driven architecture. Boston: Addison Wesley.

Menaut JC, César J. 1979. Structure and primary productivity of Lamto savannas, Ivory Coast. Ecology 60:1197-210.

Minar N, Burkhart R, Langton C, Askenazi M. 1996. The Swarm simulation system: a toolkit for building multi-agent simulations. Santa Fe: Santa Fe Institute.

Möbius K. 1877. Die Auster und die Austernwirtschaft. Berlin: Wiegandt, Hempel \& Parey.

Morin E. 1986. La méthode - 3. Paris: La connaissance de la connaissance. Seuil.

Müller JP. 2004. Emergence of collective behaviour and problem solving. In: Omicini A, Petta P, Pitt J, Eds. ESAW 2003, Engineering societies in the agents world iv: 4th international workshop (London). New York: Springer.

Naeem S, Thompson LJ, Lawler SP, Lawton JH, Woodfin RM. 1994. Declining biodiversity can alter the performance of ecosystems. Nature 368:734-7.

North MJ, Macal CM. 2007. Managing business complexity. Discovering strategic solutions with agent based modeling and simulation. Oxford: Oxford University Press.

North MJ, Tatara E, Collier NT, Ozik J. 2007. Visual agent-based model development with Repast Simphony. In: Proceedings of the agent 2007 conference on complex interaction and social emergence. Argonne: Argonne National Laboratory.

O'Neill RV. 2001. Is it time to bury the ecosystem concept? (with full military honors, of course). Ecology 82:3275-84.

O'Neill RV, DeAngelis D, Waide J, Allen TFH. 1986. A hierarchical concept of ecosystems. Princeton: Princeton University Press. 
Odum EP. 1977. The emergence of ecology as a new integrative discipline. Science 195:1289-93.

Odum EP, Odum HT. 1953. Fundamentals of ecology. Philadelphia: WB Saunders.

Pacala S, Deutschman D. 1995. Details that matter: the spatial distribution of individual trees maintains ecosystem function. Oikos 74:357-65.

Parrott L. 2005. Quantifying the complexity of simulated spatiotemporal population dynamics. Ecol Complex 2:175-84.

Pickett STA, Cadenasso ML. 2002. The ecosystem as a multidimensional concept: meaning, model, and metaphor. Ecosystems 5:1-10.

Pimm SL. 1984. The complexity and stability of ecosystems. Nature 307:321-6.

Ratzé C, Gillet F, Müller JP, Stoffel K. 2007. Simulation modelling of ecological hierarchies in constructive dynamical systems. Ecol Complex 4:13-25.

Reuter H, Holker F, Middelhoff U, Jopp F, Eschenbach C, Breckling B. 2005. The concepts of emergent and collective properties in individual-based models-summary and outlook of the Bornhoved case studies. Ecol Model 186:489-501.

Ricotta C. 2000. From theoretical ecology to statistical physics and back: self-similar landscape metrics as a synthesis of ecological diversity and geometrical complexity. Ecol Model 125:245-53.

Shlaer S, Mellor SJ. 1988. Object-oriented systems analysis. Modelling the world in data. Englewood Cliffs (NJ): Yourdon Press.
Silva Parreiras F, Staab S. 2010. Using ontologies with UML class-based modeling: the TwoUse approach. Data Knowl Eng 69:1194-207.

Simioni G, Gignoux J, Le Roux X. 2003. Tree layer spatial structure can affect savanna production and water budget: results of a 3D model. Ecology 84:1879-94.

Smith B, Prentice IC, Sykes MT. 2001. Representation of vegetation dynamics in the modelling of terrestrial ecosystems: comparing two contrasting approaches within European climate space. Global Ecol Biogeogr 10:621-37.

Tansley AG. 1935. The use and abuse of vegetational concepts and terms. Ecology 16:284-307.

Villa F. 2001. Integrating modelling architecture: a declarative framework for multi-paradigm, multi-scale ecological modelling. Ecol Model 137:23-42.

Watts DJ, Strogatz SH. 1998. Collective dynamics of 'smallworld' networks. Nature 393:440-2.

Wilensky U. 1999. NetLogo: http://ccl.northwestern.edu/netlogo/. Center for Connected Learning and Computer-Based Modeling, Northwestern University, Evanston.

Zeigler B, Kim TG, Praehofer H. 2000. Theory of modeling and simulation: integrating discrete event and continuous complex dynamic systems. New York: Academic Press.

Zhang HY, Wu JG. 2002. A statistical thermodynamic model of the organizational order of vegetation. Ecol Model 153:69-80.

Zucker JD. 2003. A grounded theory of abstraction in artificial intelligence. Philos Trans R Soc Lond Ser B 358:1293-309. 